\title{
Pregnancy-induced atypical haemolytic uraemic syndrome
}

\author{
Authors: Gwenno M Edwards, ${ }^{A}$ Jessica Notzing, ${ }^{A}$ Paul Frost ${ }^{B}$ and Rhodri Pyart ${ }^{C}$
}

KEYWORDS: pregnancy, atypical haemolytic uraemic syndrome

DOI: 10.7861/clinmed.2021-0301

\section{Case presentation}

A 19-year-old woman, previously fit and well, was admitted 4 days postpartum with a 2-day history of headache, abdominal pain and vomiting. She denied diarrhoea. Her family history was remarkable for her father having lupus and immune thrombocytopenia.

This was her first pregnancy and she delivered at term by normal vaginal delivery. She suffered a minor postpartum haemorrhage $(800 \mathrm{~mL})$, but recovered well. Rising proteinuria (urine protein/ creatinine ratio $382 \mathrm{mg} / \mathrm{mmol}$ ) was noted toward the end of the third trimester, but she remained normotensive throughout and had an otherwise unremarkable pregnancy.

On assessment, she was hypertensive at $156 / 92 \mathrm{mmHg}$. She was oligoanuric. Neurological examination demonstrated subtle generalised hyperreflexia.

She was found to have a severe acute kidney injury (AKI) associated with a microangiopathic haemolytic anaemia. Her creatinine was $528 \mu \mathrm{mol} / \mathrm{L}$ from a baseline of $53 \mu \mathrm{mol} / \mathrm{L}$. Her platelet count was low at $25 \times 10^{9} / \mathrm{L}$, with a haemoglobin $(\mathrm{Hb})$ of $48 \mathrm{~g} / \mathrm{L}$ and schistocytes seen on the blood film. Her coagulation screen was normal. Lactate dehydrogenase (LDH) was markedly elevated at 4,836 U/L. Liver function tests demonstrated elevation of bilirubin at $30 \mu \mathrm{mol} / \mathrm{L}$, with an alanine transaminase (ALT) of $55 \mathrm{U} / \mathrm{L}$ and an aspartate transaminase (AST) of $105 \mathrm{U} / \mathrm{L}$.

\section{Diagnosis}

The most likely diagnosis was initially thought to be either haemolysis, elevated liver enzymes and low platelet count (HELLP) syndrome or postpartum pre-eclampsia with severe features, given the abnormal liver function tests, the hyperreflexia and the timeline of events.

Other potential differential diagnoses included thrombotic thrombocytopenic purpura (TTP), atypical haemolytic uraemic syndrome (aHUS), retained products of pregnancy and

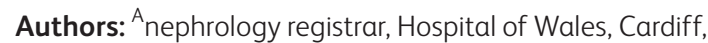
UK; ${ }^{B}$ critical care consultant, Hospital of Wales, Cardiff, UK; Cnephrology consultant, Hospital of Wales, Cardiff, UK postpartum haemorrhage. The other less likely diagnoses included systemic lupus erythematosus and antiphospholipid syndrome. It is also possible to get thrombotic microangiopathy (TMA) secondary to malignant hypertension of any cause. ${ }^{1}$

\section{Initial management}

The patient was transferred to the intensive care unit for blood pressure control with intravenous labetalol. Pending her ADAMTS13 level result, she was commenced on plasma exchange for possible TTP. Continuous veno-venous haemofiltration was started due to oligoanuric AKI. She was also given intravenous magnesium sulphate for presumed pre-eclampsia.

Her ADAMTS13 result returned as normal at 43\%, excluding a diagnosis of TTP. Escherichia coli 0157 stool culture was negative, discounting a diagnosis of Shiga toxin-producing $E$ coli haemolytic uraemic syndrome (STEC-HUS). Immunology was negative, including normal antiphospholipid antibody screen and normal antinuclear antibody.

Given the above results, we felt that the patient should commence eculizumab for possible pregnancy aHUS. Her case was promptly discussed with the National Renal Complement Therapeutics Centre in Newcastle upon Tyne, who agreed that we had to consider it as a possible cause of the presentation. The centre's approval is required to obtain eculizumab in the UK and they have a $24 / 7$, consultant-led on-call service. Details can be found on their website at www.atypicalhus.co.uk/emergencyreferrals. Within 24 hours she had received her first dose of eculizumab. She was also commenced on prophylactic antibiotics and vaccinated against meningococcus.

\section{Progression and outcome}

Following the first dose of eculizumab, the patient's blood pressure normalised and she was transferred to the nephrology ward. Her platelet count rapidly climbed to normal levels within days (Fig 1).

She remained dialysis dependent and anuric; therefore, a tunnelled dialysis catheter was inserted. The patient declined a renal biopsy due to the risk of further delay in getting home to her baby.

Remarkably, 4 weeks later, her renal function had almost completely recovered (creatinine of $86 \mu \mathrm{mol} / \mathrm{L}$ ), which allowed the discontinuation of dialysis. Her blood pressure was $117 / 77 \mathrm{mmHg}$ on no treatment.

Further communication with Newcastle led to genetic complement analysis, revealing a complement factor $\mathrm{H}$ (CFH) mutation - c.182delA p.(Asn61fs) - for which she is currently 


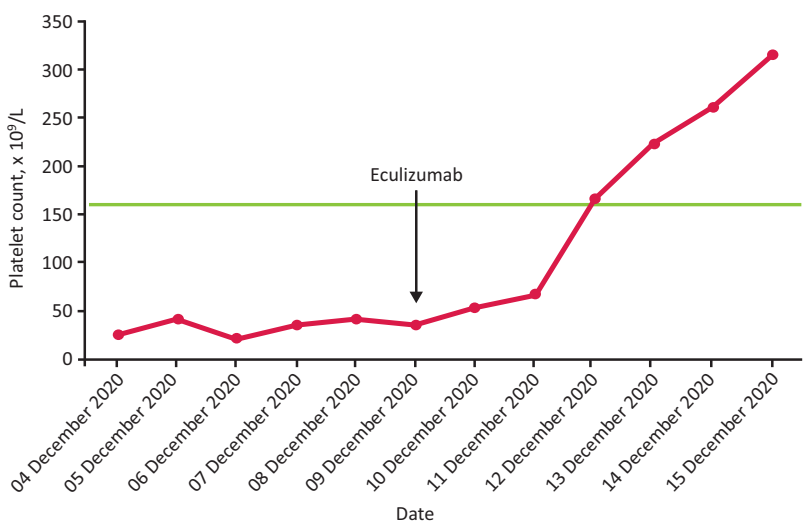

Fig 1. Platelet count progression before and after eculizumab administration. The green line indicates the lower end of the normal range.

maintained on fortnightly outpatient eculizumab infusions. Her family are being counselled on genetic testing.

\section{Discussion}

TMA is a group of disorders characterised by a triad of intravascular microangiopathic haemolytic anaemia, thrombocytopenia and vascular thrombosis. ${ }^{2}$ In the postpartum period, TMA is usually found in association with HELLP syndrome or pre-eclampsia, but can rarely be due to pregnancy aHUS or TTP. ${ }^{3}$

Due to similar features, differentiating between aHUS/TTP and pre-eclampsia or HELLP syndrome can be difficult, but an open mind is required, guided by clinical clues (Table 1). Making the correct diagnosis is critical, as there are specific therapies for TTP and aHUS which need urgent initiation (Table 1). ${ }^{3}$ The challenge

\section{Table 1. Differentiating clinical signs between} atypical haemolytic uraemic syndrome and preeclampsia / haemolysis, elevated liver enzymes and low platelet count syndrome

$\begin{array}{lll}\text { Diagnosis } & \text { Clinical clues } & \text { Main treatment } \\ \text { aHUS } & \text { Creatinine }>200 \mu \mathrm{mol} / \mathrm{L} & \begin{array}{l}\text { Eculizumab } \\ \mathrm{LDH}>1,000 \mathrm{U} / \mathrm{L}\end{array} \\ & \mathrm{Hb}<80 \mathrm{~g} / \mathrm{L} & \\ & \begin{array}{l}\text { Persistence }>72 \text { hours } \\ \text { postpartum }\end{array} & \\ & \text { Creatinine }<200 \mu \mathrm{mol} / \mathrm{L} & \text { Delivery } \\ \text { Pre-eclampsia / } & \mathrm{LDH}<1,000 \mathrm{U} / \mathrm{L} & \\ \text { HELLP } & \mathrm{Hb}>110 \mathrm{~g} / \mathrm{L} & \\ \text { syndrome } & \text { Resolution within } 72 \text { hours } & \\ & \text { of delivery } & \end{array}$

aHUS = atypical haemolytic uraemic syndrome; $\mathrm{Hb}=$ haemoglobin; HELLP = haemolysis, elevated liver enzymes and low platelet count; LDH = lactate dehydrogenase. with aHUS is that a definitive diagnosis through genetic testing takes many weeks. Treatment, which is expensive and (in the UK) requires approval from Newcastle, cannot be delayed during this time.

aHUS is a rare, genetically determined dysregulation of the alternative pathway of the complement cascade. A trigger to activate complement is required to manifest the disease, with pregnancy and delivery both being powerful triggers. ${ }^{4}$ Our patient had a CFH mutation. CFH is a plasma protein that regulates the alternative pathway. Over 120 CFH mutations have been identified in patients with aHUS. ${ }^{5}$

Treatment of aHUS involves complement blockade. Eculizumab is a recombinant monoclonal antibody that inhibits terminal complement activation at the $\mathrm{C} 5$ protein and thereby reduces haemolysis and TMA. Treatment is potentially lifelong, although research is underway into the risk of relapse on treatment cessation. Patients require vaccination against Neisseria meningitidis and prophylactic antibiotics. ${ }^{6}$

\section{Key points}

> TMA in pregnant or postpartum women is potentially a lifethreatening condition.

$>$ Where clinically suspected, the priority is to exclude TTP and (in the UK) to have timely discussion with Newcastle to streamline investigations and allow prompt treament with eculizumab.

> In complex or critical cases, we recommend a multidisciplinary team discussion involving critical care, haematology, obstetrics and nephrology to expedite diagnosis and treatment, which could be life-saving.

> Early treatment with eculizumab was critical in our patient's renal recovery.

\section{References}

1 Khanal N, Dahal S, Upadhyay S, Bhatt VR, Bierman PJ. Differentiating malignant hypertension-induced thrombotic microangiopathy from thrombotic thrombocytopenic purpura. Ther Adv Hematol 2015:6:97-102.

2 George JN, Nester CM. Syndromes of thrombotic microangiopathy. N Engl J Med 2014;371:654-66.

3 Gupta M, Feinberg BB, Burwick RM. Thrombotic microangiopathies of pregnancy: Differential diagnosis. Pregnancy Hypertens 2018:12:29-34.

4 Kaartinen K, Martola L, Aaltonen S, Meri S. Diagnostic dilemma of thrombotic microangiopathy in pregnancy. Kidney Int Rep 2020:6:529-33.

5 Feehally J, Floege ], Tonelli M, Johnson RJ (eds). Comprehensive clinical nephrology, 6th edn. Elsevier, 2019.

6 Joint Formulary Committee. British National Formulary. London: BMJ Group and Pharmaceutical Press. www.medicinescomplete. com [Accessed on 10 June 2021].

Address for correspondence: Dr Gwenno Mair Edwards, Department of Nephrology, University Hospital of Wales, Heath Park, Cardiff CF14 4XW, UK.

Email: gwenno.m@hotmail.co.uk 